\title{
Complex dynamical networks constructed with fully controllable nonlinear nanomechanical oscillators
}

Warren Fon, Matthew Matheny, Jarvis Li, Lev Krayzman, Michael

Cross, Raissa M D'Souza, James Crutchfield, and Michael Lee Roukes

Nano Lett., Just Accepted Manuscript • DOI: 10.1021/acs.nanolett.7b02026 • Publication Date (Web): 08 Sep 2017

Downloaded from http://pubs.acs.org on September 8, 2017

\section{Just Accepted}

"Just Accepted" manuscripts have been peer-reviewed and accepted for publication. They are posted online prior to technical editing, formatting for publication and author proofing. The American Chemical Society provides "Just Accepted" as a free service to the research community to expedite the dissemination of scientific material as soon as possible after acceptance. "Just Accepted" manuscripts appear in full in PDF format accompanied by an HTML abstract. "Just Accepted" manuscripts have been fully peer reviewed, but should not be considered the official version of record. They are accessible to all readers and citable by the Digital Object Identifier (DOI®). "Just Accepted" is an optional service offered to authors. Therefore, the "Just Accepted" Web site may not include all articles that will be published in the journal. After a manuscript is technically edited and formatted, it will be removed from the "Just Accepted" Web site and published as an ASAP article. Note that technical editing may introduce minor changes to the manuscript text and/or graphics which could affect content, and all legal disclaimers and ethical guidelines that apply to the journal pertain. ACS cannot be held responsible for errors or consequences arising from the use of information contained in these "Just Accepted" manuscripts. 


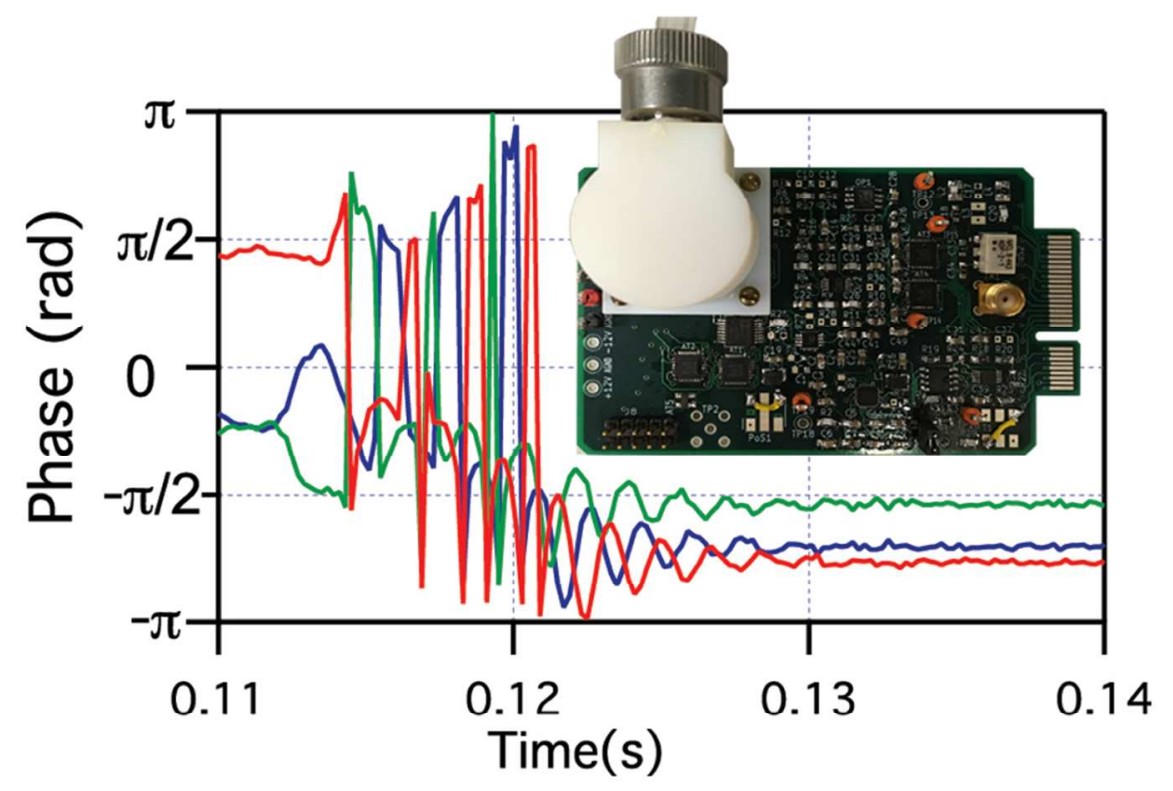

$96 \times 66 \mathrm{~mm}(276 \times 276 \mathrm{DPI})$ 


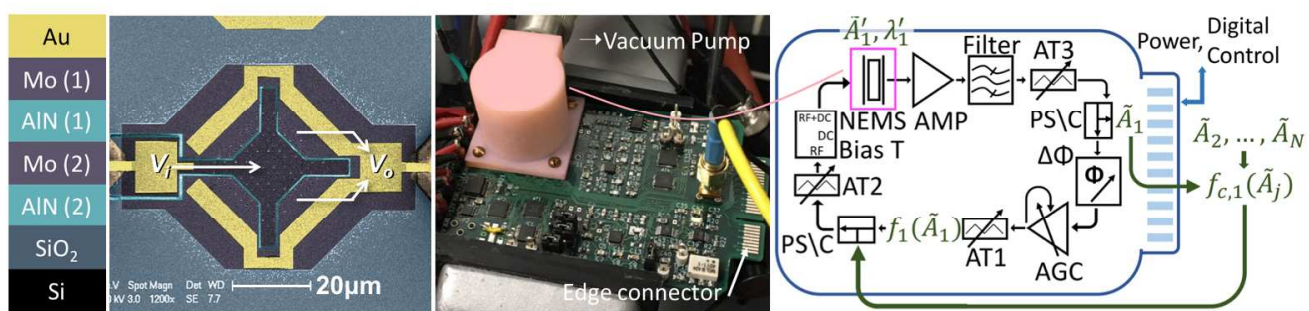

Figure 1: Modular nonlinear NEMS oscillator. Left panels: Device layer stack and a colorized scanning electron micrograph showing a top view of an aluminum nitride (AIN) NEMS resonator. The input and output thin-film molybdenum (Mo (1)) electrodes (dark purple) are electrically separated by an etched trench that exposes the underlying AIN (1) (turquoise). Thin-film gold electrodes (yellow) provide contacts to Mo electrodes; the bottom Mo (2) layer forms the counter-electrode. The dotted violet line approximately delineates the extent of the membrane resonator. Right panels: The oscillator PCB and its functional block diagram. The NEMS resonator is housed in a small vacuum chamber (colorized pink). Voltage biases, oscillator waveforms, and digital signals are accessible from either the edge connector and PCB test points or via SMA connectors.

$177 \times 44 \mathrm{~mm}(278 \times 278$ DPI $)$ 

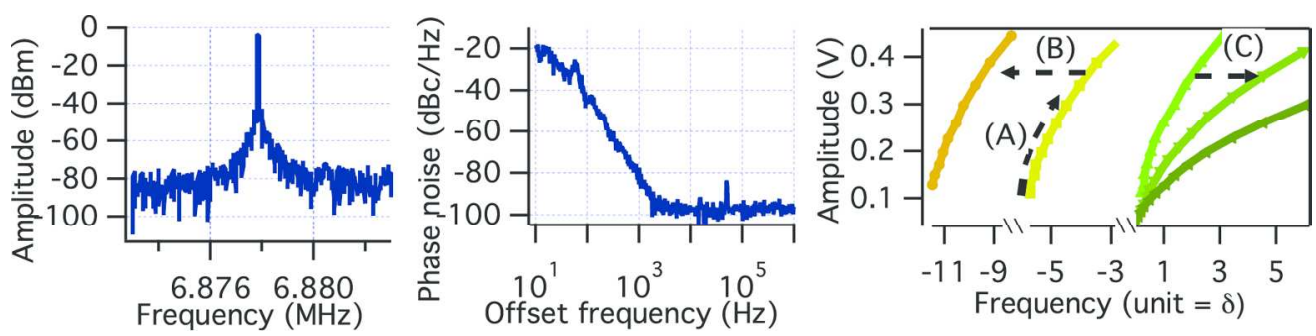

Figure 2. Signal analysis of modular NEMS PCB oscillator. Power spectrum (left) and the phase noise (middle). The power spectrum shows a dominant peak near the NEMS resonant frequency. On the right is the amplitude frequency of an oscillator node under different configuration parameters. In (A), the feedback drive is increased by reducing attenuator AT1 to form the "backbone" curve characteristic to the Duffing nonlinearity. In (B), VDC $=1 \mathrm{~V}$ is applied to tune by $\sim$. In (C), AT2 and AT3 are changed by equal and opposite amounts, which increases the apparent nonlinearity while keeping amplitude constant.

$166 \times 40 \mathrm{~mm}(300 \times 300$ DPI $)$ 

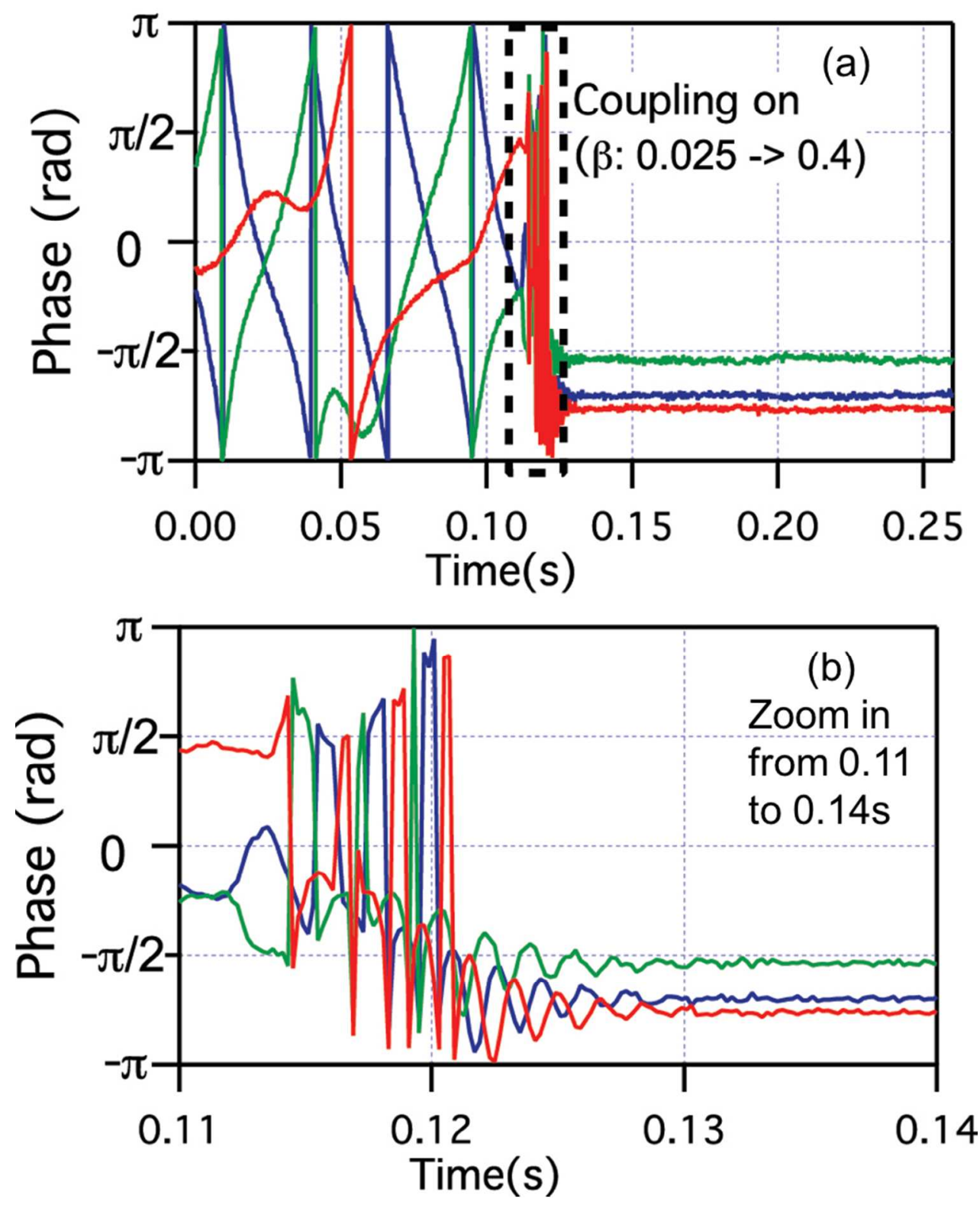

Figure 3: The time-continuous phase difference of 3 oscillators configured as a ring network. On the top panel (a), at $t=0 s$, before the coupling is turned on, the nodes are configured to have and for each oscillator and are individually tuned to attain a frequency difference of . At $t=0.115 \mathrm{~s}$, coupling is switched on. This induces oscillator synchronization after $\sim 15 \mathrm{~ms}$. The detailed evolution of the oscillators during the synchronization process (black dashed box in top panel) is fully captured and displayed in the lower panel

(b).

$89 \times 110 \mathrm{~mm}(259 \times 259 \mathrm{DPI})$ 


\title{
Complex dynamical networks constructed with fully controllable nonlinear nanomechanical oscillators
}

Warren Fon, ${ }^{1 \ddagger}$ Matthew H. Matheny, ${ }^{1 \ddagger}$ Jarvis $\mathrm{Li}^{1}{ }^{1}$ Lev Krayzman, ${ }^{1}$ Michael C. Cross, ${ }^{1}$ Raissa M. D'Souza, ${ }^{2,4}$ James P. Crutchfield, ${ }^{3,4}$ and Michael L. Roukes ${ }^{1 *}$

1 Condensed Matter Physics and Kavli Nanoscience Institute, California Institute of Technology, Pasadena, California 91125, United States

2 Complexity Sciences Center, Department of Computer Science, and Department of Mechanical and Aerospace Engineering, University of California, Davis

3 Complexity Sciences Center and Department of Physics, University of California, Davis

4 Santa Fe Institute, 1399 Hyde Park Rd, Santa Fe, NM 87501

\begin{abstract}
Control of the global parameters of complex networks has been explored experimentally in a variety of contexts. Yet, the more difficult prospect of realizing arbitrary network architectures, especially analog physical networks, that provide dynamical control of individual nodes and edges has remained elusive. Given the vast hierarchy of timescales involved, it also proves challenging to measure a complex network's full internal dynamics. These span from the fastest nodal dynamics to very slow epochs over which emergent global phenomena, including network synchronization and the manifestation of exotic steady states, eventually emerge. Here, we demonstrate an experimental system that satisfies these requirements. It is based upon modular, fully controllable, nonlinear radio-frequency nanomechanical oscillators, designed to form the nodes of complex dynamical networks with edges of arbitrary topology. The dynamics of these oscillators and their surrounding network are analog, continuous-valued and can be fully interrogated in real time. They comprise a piezoelectric nanomechanical membrane resonator, which serves as the frequency-determining element within an electrical feedback circuit. This embodiment permits network interconnections entirely within the electrical domain, and provides unprecedented node and edge control over a vast region of
\end{abstract}


parameter space. Continuous measurement of the instantaneous amplitudes and phases of every constituent oscillator node are enabled, yielding full and detailed network data without reliance upon statistical quantities. We demonstrate operation of this platform through the real-time capture of the dynamics of a 3-node ring network as it evolves from the uncoupled state to full synchronization.

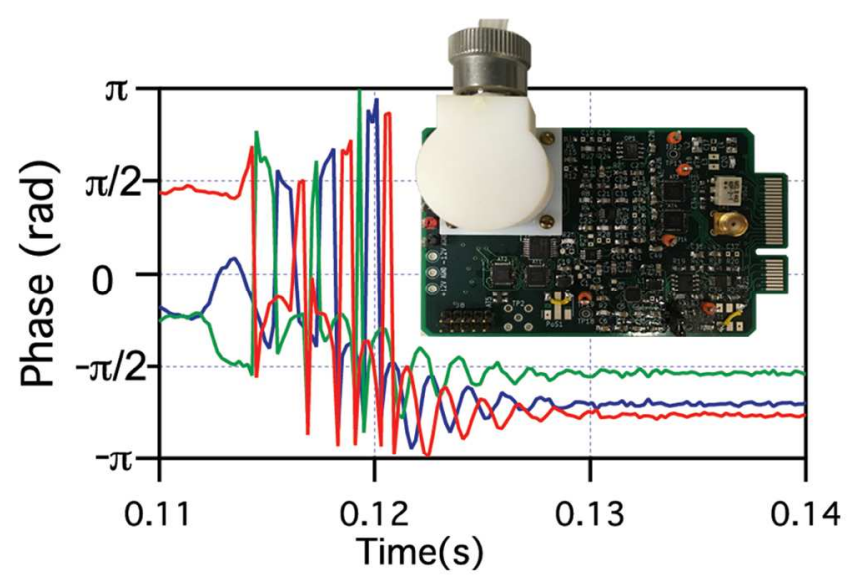

KEYWORDS: NEMS, Complex Networks, Nanomechanical Oscillators, Nonlinearity, Dynamical Systems, Control, Synchronization 
A variety of natural systems serve as resources for empirical studies in network science; these include neural networks ${ }^{1}$, gene regulatory networks ${ }^{2}$, infrastructure networks ${ }^{3-5}$, and social networks ${ }^{6-8}$. In most cases, experimentalists have limited ability to configure and manipulate the individual nodes and edges of such systems. Experimental studies of macroscopic network behavior, in which local manipulations can be performed at the level of individual nodes and edges, are of significant and general interest. Many physical systems - including arrays of Josephson junctions $^{9}$, nanoscale spin-torque oscillators $(\mathrm{STOs})^{10,11}$, mechanical clocks ${ }^{12}$, optical parametric oscillators $^{13}$, optomechanical resonators $(\mathrm{OMRs})^{14,15}$, optoelectronic resonators ${ }^{16,17}$, and digital-delay oscillators based on finite-state feedback shift registers built using fieldprogrammable-gate-arrays (FPGAs) ${ }^{18}$ - have been interconnected to form networks. While these systems are based on intriguing physical phenomena, an optimal physical platform for network exploration must not only permit realization of arbitrarily configurable network graphs $^{19}$, they must also facilitate the complete control and readout of these individual nodes and edges with high fidelity at full bandwidth. However, upscaling such platforms to realize complex networks comprising 10 to 100 nodes and beyond with arbitrary connection topologies can be both technologically challenging and expensive. An important additional consideration is the fundamental timescale for the fastest dynamics set by the nodal oscillation frequency. This should be neither too slow nor too fast, as it controls the interval over which very slowly-emerging collective network phenomena - associated with the evolution with exotic or weakly stable states - become fully manifested.

For the aforementioned reasons, recent experimental realizations of synchronized networks of coupled oscillators are typically non-ideal. We consider two cases. First, nanoscale STOs and 
Josephson junctions typically operate at 10's to 100 's of GHz; this makes direct observation of real-time dynamics, such as phase slipping, challenging ${ }^{20}$. Additionally, the coupling between STOs in experiments to date is determined by their spatial proximity. Hence, network edge topology is primarily determined by geometry, and is thus subject to limitations imposed by fabrication technology. Second, realizing synchronization within networks of OMRs, poses special challenges. Here it is critical to draw the distinction between synchronization and mode hybridization. Synchronization occurs solely in a system of weakly-coupled, independent oscillators - each of which has, in the limit of vanishing inter-oscillator coupling, complete phase freedom. ${ }^{20}$ Implementing a multiplicity of independent optomechanical oscillators with the requisite phase freedom thus necessitates use of a dedicated optical pump for each node. The inter-oscillator coupling must then be implemented independently from these internal, "nodal" components. To our knowledge, independence between optomechanical nodes and inter-oscillator coupling has only been achieved with inter-nodal coupling mediated in the electrical, rather than the optical, domain ${ }^{21}$. In other recent optomechanical experiments that have purported to manifest synchronization, ${ }^{22,}{ }^{23}$ the optical source used to drive the parametric nodal oscillations also provides common feedback to all resonators in the array. With this implementation, one source is employed to induce both the inter-node coupling and excitation of the resonators. Here, the strong coupling between the nodal resonators, and the lack of phase independence between them, yields hybridization of resonator modes rather than effecting synchronization amongst independent oscillators. In this work, we employ separate feedback loops for each oscillator and then couple them by independent means within the electrical domain. Synchronization between independent, weakly-coupled oscillators is the 
basis of the Kuramoto model ${ }^{24}$ and is essential, for example, for proposals to use oscillator networks as elements of associative memory in the application of for pattern recognition. ${ }^{25}$

For the reasons outlined, upscaling to large networks is infeasible with the embodiments described above. By contrast, recently developed FPGA-based digital logic oscillators are indeed cost-effective to scale up, as a single FPGA chip can instantiate hundreds of oscillators. ${ }^{18}$ The limitation in this case is that FPGA network realizations are digital and binary; hence, they are restricted to the domain of phase oscillators, that is rotators. These do not exhibit the full range of oscillator behavior that includes both phase and amplitude dynamics. ${ }^{18}$ For this reason, FPGA-based networks facilitate only specific explorations of the subset of synchronization dynamics involving Kuramoto-like behavior. ${ }^{26}$ Accordingly, our work here complements FPGAtype oscillators in the sense that, although initial scaling with NEMS oscillators is costlier, a much wider range of network topologies and dynamics becomes accessible.

In this Letter, we describe a new model network system implemented with modular, radio frequency, electromechanical oscillator "nodes" that are based upon piezoelectric nanoelectromechanical systems (NEMS). To form fully controllable, synchronized oscillator networks with arbitrary topology, we designed this system employing our previously-developed circuit topology that enables electrical coupling of two NEMS nodes. ${ }^{27}$ Here, we realize a modular, self-contained oscillator node that can be used to significantly extend the previous work. In our ongoing work, these oscillator nodes will be assembled to form large networks, from 10 to 100 nodes, with arbitrarily configured edges. 
We model our oscillator network, which comprises an ensemble of high quality factor, weakly coupled, nonlinear mechanical resonators as a set of saturated, nonlinear (Duffing) oscillators ${ }^{28}$. Using scaled amplitude, time, and frequency variables; we obtain an equation of motion for the normalized complex nodal amplitudes, $A_{j}$ :

$\frac{d A_{j}}{d T}-i\left(\frac{\delta_{j}}{2}+\alpha_{j}\left|A_{j}\right|^{2}\right) A_{j}+\frac{A_{j}}{2}=\frac{1}{2} e^{i \phi_{j}}+f_{c, j}\left(A_{1}, \cdots, A_{N}\right)$.

Here, $j$ is the oscillator node index spanning from 1 to $N$, and $N$ is the total number of nodes. We normalize time, $t$, by the energy decay rate, $T=2 \pi f_{a} t / Q$; it is over this (slow) timescale that the coupled dynamics of the network evolve. $f_{a}$ is the average linear (small amplitude) frequency of all oscillator nodes, $Q$ is the quality factor of the resonators (identical in all nodes). The set of normalized oscillator frequencies, which manifests frequency dispersion over the slow time scale, are defined as $\delta_{j}=Q\left(\frac{f_{j}^{2}}{f_{a}^{2}}-1\right) \sim 2 Q \frac{f_{j}-f_{a}}{f_{a}}$. These are the normalized offset between the $j$-th oscillator's frequency, $f_{j}$, and the frequency average, $f_{a}$, such that $\sum_{i} \delta_{i}=0$. The use of normalized time and frequency also effectively normalized the damping of the resonator as represented by the last term of the L.H.S. of eq. (1). The right-hand side of the equation represents the drives to the system: The feedback function to locally sustain the oscillation can be arbitrarily complex, but for our current system we use a saturated nonlinear function $f_{f, j}\left(A_{j}\right)=\frac{1}{2} e^{i \phi_{j}}$ where $\phi_{j}=\operatorname{Arg}\left(A_{j}\right)$ is oscillation phase. The saturation amplitude is identical to all oscillator nodes. In Eq. 1, it appears to have constant amplitude solely due to the scaling of the equation. In the experimental calibration of the oscillator nodes, each node is decoupled from the network and then first driven at low (linear) amplitude to yield linear frequency $\delta_{j}$. Then, the feedback drive is then raised to the desired frequency detuning $\alpha_{j}$. The 
experimentally observed amplitude will also rise but it simply scales the model amplitude to unity, i.e., $\left|A_{j}\right|=1$ corresponds to observed amplitude when the oscillator is decoupled from the network. Note that the relevance of the oscillation amplitude to the dynamics lies with its detuning to the frequency. Finally, $f_{c, j}\left(A_{1}, \cdots, A_{N}\right)$ is the coupling between oscillators defined by network topology such as the ring network as discussed below. The coupling discussed in this work is linear in amplitude. Thus, the functional form of the coupling applied both to the scaled and experimental measured amplitude. However, there is modification in phase of the feedback to produce so-called reactive or dissipative coupling. In a companion theoretical analysis, we describe the rich attractor switching dynamics exhibited by oscillator networks comprising from 3 to over 100 nodes. ${ }^{29}$ Here, we demonstrate the detailed construction of NEMS oscillator nodes to illustrate the flexibility of our system.

In network analysis, it is common to study the effect of network topology upon the distribution of the oscillator's linear frequencies $\left(\delta_{j}\right)$. In most experimental systems, the initial, linear oscillator (nodal) frequencies are pre-determined and are either not tunable, or are adjustable only over a limited range. Here, our platform allows precise and arbitrary specification of $\delta_{j}$ for each node. In our instantiation, nodal oscillation frequencies range from 1 to $20 \mathrm{MHz}$; the associated "slow time" scale, $T$, which dictates the interval over which network steady states evolve, is of order milliseconds. Accordingly, the complete state of the network, which is characterized by the complex- and continuous-time- valued oscillation $A_{j}$ (including the amplitude and phase and of each node), can easily be captured with high fidelity. Further, networks built from these oscillator nodes can be configured with arbitrary network topologies (for example, with variations in the strength of coupling between neighbors), and then set into 
motion with arbitrarily complex initial conditions (for example, by locking the nodes with external references). Subsequently, network state reconfigurations can be achieved within submillisecond time scales, enabling acquisition of thousands of traces displaying slow network evolution in under one hour. These features - especially the ability to capture the detailed evolution of all nodes with high fidelity and rapid topological reconfiguration - are unprecedented features of our system.

Studies of abrupt modifications are key to modeling and understanding prototype networks designed to mimic real-world systems. Realizing abrupt changes to network topology requires imposing perturbations faster than the slow-time scale of the oscillator node. For example, we can abruptly isolate a single or multiple oscillators nodes (setting $f_{c, j}\left(A_{1}, \cdots, A_{N}\right)=0$ for a subset of nodes) to observe the effect upon network dynamics. Other abrupt modifications achievable in real time include tuning of individual or multiple nodal parameters (for example, $\alpha_{j}, \delta_{j}$, the phase of feedback function $f_{f, j}\left(A_{j}\right)$, etc.) and also permit local injection of noise into the network.

Over the past three decades, micro- and nano- electromechanical systems (MEMS/NEMS) have engendered an evolution of clock architectures from their previous, macroscale, hand assembled realizations to their contemporary embodiments as micro- and nanoscale electromechanical devices produced en masse by foundry-scale methods. The dynamics of individual MEMS and NEMS oscillators ${ }^{28}$ are now well understood both theoretically and experimentally. To date, however, only a few investigations of coupled networks of NEMS or MEMS oscillators have been reported. ${ }^{29}$ Purely mechanical coupling between mechanical resonators, such as Huygen's historic clock synchronization ${ }^{12}$, is typically weak and difficult to 
tune. However, for MEMS and NEMS resonators, it is precisely this weak mechanical coupling to their surroundings that yields the benefit of their remarkably low dissipation rates. In this work, we desire to preserve the low dissipation of NEMS elements while realizing arbitrarily large and precisely controllable coupling between oscillator nodes within network topologies. To this end, we first engineer efficient conversion of nanomechanical motion into electrical signals via the piezoelectric effect. Subsequently, large electromechanical node-to-node coupling is easily attained within the electrical domain.

An important attribute of NEMS, as compared with other resonators - including those patterned from quartz crystals, or those based on lasers, biological or chemical systems, or MEMS - is their ability to be easily driven well past their linear dynamic range to controlled and strongly nonlinear response. With NEMS, a Duffing nonlinearity arises purely within the mechanical domain due to the onset of amplitude-dependent tension; this causes a deviation from the harmonic elastic potential existing for small displacements. In the nonlinear regime, the amplitude and phase of the oscillations become dynamically coupled.

The NEMS we employ in this work resonate at radio frequencies ( 1 to $20 \mathrm{MHz}$ ) with high quality factors ( $Q=1000$ to 10000 in vacuum). The coupling between two oscillators is considered strong when the coupling function is at the order of the oscillation amplitude, which leads to resonator mode hybridization. For example, strong coupling between two oscillators occurs when $f_{c, 1}=-f_{c, 2}=\frac{1}{2}\left(A_{1}-A_{2}\right)$. In our experiment of network synchronization, nevertheless, only weak coupling is warranted. Likewise, we normalize the time and nonlinearity-induced frequency shift to the natural linewidth of the resonators at low oscillator amplitudes (where the nonlinear stiffness of the resonator is not observed). Thus, for example, if the resonator has 
frequency $f=2 \mathrm{MHz}, Q=2000$, and a frequency detuning $\Delta f=1 \mathrm{kHz}$, then a unit of slow time corresponds to $1 \mathrm{~ms}$ in real time $(T=1 \rightarrow t=1 \mathrm{~ms})$ and the normalized frequency shift is $\frac{\Delta \delta}{2}=1$

The piezoelectric resonator provides efficient mechanical-to-electrical transduction with a direct forward transmission coefficient, $S_{21} \sim-27 \mathrm{~dB}$, and a signal-to-background ratio $>30 \mathrm{~dB}$. These permit stable locking of the oscillation in feedback loops constructed with off-the-shelf integrated circuits (ICS). Displacement transducers and actuators that couple mechanical motion into electrical signals, and vice-versa, facilitate straightforward coupling between nodes with network edges implemented solely within the electrical domain. An outstanding feature of our piezoelectric NEMS resonators is the ability to directly tune their vibrational frequencies, $\delta_{j}$, by application of a DC voltage. ${ }^{30}$ Piezoelectric NEMS provide a very wide tuning range, $\Delta \delta_{j} \sim \pm$ 5; such a large frequency span is rarely achieved with other physical systems.
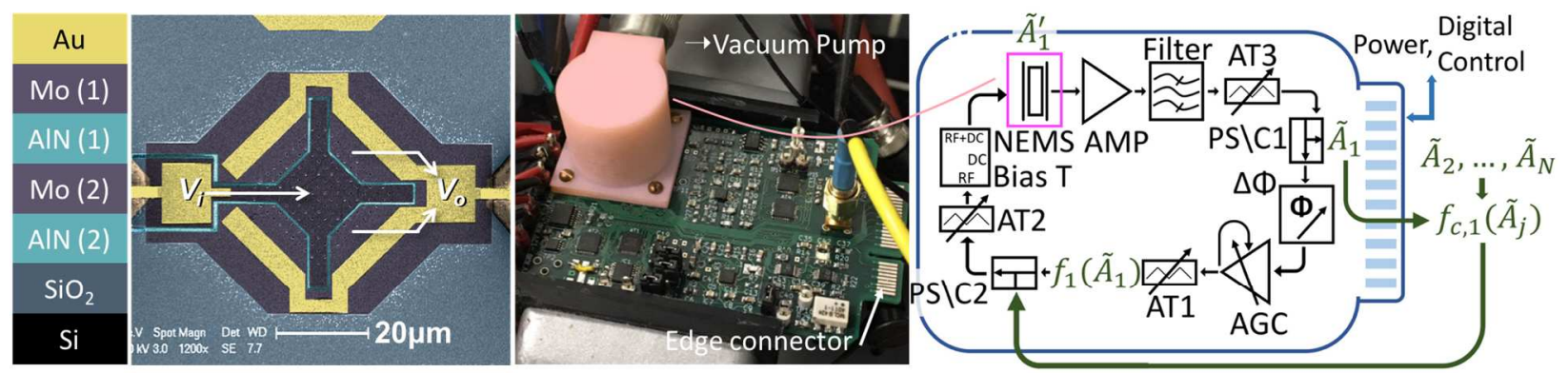

Figure 1: Modular nonlinear NEMS oscillator. Left panels: Device layer stack and a colorized scanning electron micrograph showing a top view of an aluminum nitride (AIN) NEMS resonator. The input and output thin-film molybdenum (Mo (1)) electrodes (dark purple) are electrically separated by an etched trench that exposes the underlying AIN (1) (turquoise). Thinfilm gold electrodes (yellow) provide contacts to Mo electrodes; the bottom Mo (2) layer forms 
the counter-electrode. The dotted violet line approximately delineates the extent of the membrane resonator. Right panels: The oscillator PCB and its functional block diagram. The NEMS resonator is housed in a small vacuum chamber (colorized pink). Voltage biases, oscillator waveforms, and digital signals are accessible from either the edge connector and PCB test points or via SMA connectors.

Our NEMS resonators are fabricated from ultrathin piezoelectric multilayer "stacks", as depicted in Figure 1. Elsewhere, we describe the piezoelectric NEMS device fabrication process $^{31,32}$. Feedback oscillators constructed from these NEMS resonators prove ideal for the study of synchronization in nonlinear oscillator networks for several reasons. The thinness (50 to $150 \mathrm{~nm}$ ) of our piezoelectric stacks permits large area- (or length/width) to-thickness ratios, even for the smallest devices. Large aspect ratios are key to achieving an early onset of nonlinearity, that is, one manifested at small displacement amplitudes. Additionally, as represented in Eq. (1), with inclusion of the nonlinear Duffing term and feedback with a controlled saturation level, the oscillator dynamics of nonlinear NEMS can be accurately modeled as a generic, self-sustained oscillator. ${ }^{33}$ Further, even with their high $Q$ 's, high frequency NEMS resonators have relatively short damping times on the order of the slow, normalized time $T$, and this leads to evolution of their coherent, 'slow-time' network-coupled dynamics over reasonable, millisecond-scale intervals. These frequencies and time scales permit high-resolution sampling of the full, real-time dynamics that can be affected by stochastic processes near the resonator frequency. 
Direct linear conversion between stress/strain and charge/field provided by the piezoelectric "stack" allows for reduced circuit complexity, as compared with other transduction schemes ${ }^{34}$ 35 . The membrane area of $\sim 500 \mu \mathrm{m}^{2}$ of our resonators provides large and robust output signals. In addition to its use for quasi-static frequency tuning, piezoelectric voltage control of resonant frequency can be employed dynamically (with AC signals) to facilitate parametric actuation. ${ }^{36}$ Electrical frequency tuning can also be used to momentarily decouple specific nodes from the network, thus providing a useful means for network manipulation and control.

In our previous demonstration of synchronized NEMS oscillators, we employed connectorized and rack-mounted electronic instrumentation to implement oscillator feedback and network coupling. ${ }^{37}$ To permit construction of more complex networks, a versatile, compact and more upscalable implementation is essential. In this work, we implement NEMS oscillators with small printed circuit board (PCB) circuitry (Figure 1). These PCB circuits are a versatile and costeffective platform for interconnection, especially for analog signals. Nonlinear NEMS oscillator networks comprising from tens to hundreds of nodes are readily achievable. The PCBs are designed with commercial software, fabricated by commercial manufacturers, and populated with ICs (providing the requisite amplification, multiplexing, saturation, attenuation and phaseshifting functions, etc.) - with total expenditure of less than $\$ 1,000$. While our entire NEMS network - including all nodes and edges - can be implemented on a single PCB, here we construct individual, modular, "plug-and-play" oscillator nodes that are connected via a "network board" comprising the network's edges that complete and define the network topology. This architecture greatly reduces the cost and design complexity and permits very flexible realization of network topologies. 
The modular oscillator nodes are constructed on credit-card-sized PCBs on which the NEMS resonator dies are mounted. Wirebonds connect the NEMS die to the module's electronic feedback circuitry. Operation in vacuum at or below $100 \mu$ bar is essential to ensure flexural mode resonances with high quality factors, as well as to ensure optimal frequency stability. We achieve this by housing the NEMS device within a miniaturized, on-board plastic vacuum enclosure, fabricated by 3D printing and attached to the PCB with small screws (Fig. 1).

To understand the oscillator node in more detail, we explain the function of the physical components depicted in Figure 1 with the mathematical model. Noting that the amplitude $A_{1}$ is scaled in equation 1 , we devote the experimental amplitude as $\tilde{A}_{1}$. The scale factor is that $A_{1}=1$ while $\tilde{A}_{1}$ is determined when the oscillator is decoupled from the network. Thus, the scale factor does change upon change amplitude of the feedback drive. The complex oscillator signal $\tilde{A}_{1}$ (with amplitude and phase) is available at the output of the power splitter/combiner, labeled PS/C1. This component (PS/C1) splits the oscillation into two equal portions: one for the connection into the network and the other to sustain the feedback for oscillation. The saturated feedback drive is generated by the combined action of the phase shifter $(\Delta \phi)$, automatic gain control amplifier (AGC) and tunable attenuator (AT1). Since the phase of oscillation is delayed by the components in the loop (and this phase shift is generally frequency dependent), we adjust the phase shift through the loop at the point of maximum amplitude where the feedback does not affect oscillator frequency. The tunable phase shifter, realized as an active all-pass filter with one terminal impedance tunable via a digital potentiometer, ${ }^{38}$ provides a phase tuning range of $\sim 150^{\circ}$ below $10 \mathrm{MHz}$. The automatic gain control (AGC) amplifier is used to clamp the output amplitude at a fixed "saturated" value, regardless of input signal level. This 
approach is commonly employed to suppress the contribution of the feedback amplifier's amplitude noise on oscillator phase noise. In the present setup, we employ a linear-in-dB variable-gain amplifier (Analog Devices AD8368) for this role; it permits setting the output amplitude through bias resistors. To achieve log scaling of the saturated output, we use a digitally-controlled variable attenuator (AT1) at the output. Like the digital potentiometer embedded in the phase shifter, AT1 is also alterable in real-time; we control it remotely through the digital port on the network board using serial peripheral interface (SPI) protocols.

After AT1, the local feedback drive is combined with coupling arriving from network edges via the power combiner PS/C2. Together, this combined drive signal, represented by the right-hand side of Eq. (1), actuates the resonator. In Figure 1, the vacuum-based NEMS resonator is evident at the left top corner of the board. Two attenuators, AT2 and AT3, at the input and output of the device, respectively, are used to condition the signals to and from the NEMS. Through the Bias-T, a DC voltage $V_{D C}=-10$ to $10 \mathrm{~V}$ can be applied to the NEMS to change the membrane stress and thereby tune the resonator's frequency. This $V_{D C}$ bias is provided by an ultra-stable 16-bit digital-to-analog converter (DAC), ensuring that frequency tuning, $\Delta \delta$, is steady and adjustable with resolution below 100ppm. To achieve optimal signal-to-noise ratio, the NEMS output signal is amplified and filtered by an amplifier chain (AMP) comprising, at its front end, an operational amplifier (op amp) based transimpedance amplifier (TIA) ${ }^{38}$ delivering a gain of 40,000V/A. The TIA was adopted for readout since it yields optimal performance with our piezoelectric transducer, which behaves as a current source. The second gain stage is a twostage non-inverting op amp. The total gain of the cascaded readout amplifier chain is $50 \mathrm{~dB}$. The effective forward scattering parameter achieved at resonance, $S_{21}$, is $\sim 25 \mathrm{~dB}$; depending upon 
operating conditions; this yields output voltages ranging from $\sim 1$ to $1000 \mathrm{mV}$. Low pass, high pass, and bandpass filters are incorporated within the amplifier chain to provide out-of-band signal suppression. As shown in Figure 2, oscillation at $\sim 6.878 \mathrm{MHz}$ is observed from a PCB NEMS oscillator node with measured phase noise of approximately $-80 \mathrm{dBc} / \mathrm{Hz}$ at $1 \mathrm{kHz}$ offset. This level of stability is comparable to the performance of monolithic nanomechanical oscillators of similar size and mass. ${ }^{39}$ The roll-off in the phase noise spectrum, roughly proportional to $\sim 1 / f^{4}$, suggests that this noise originates from environmental temperature or vibrational fluctuations. ${ }^{40}$ Although commercial oscillator

modules exist with lower phase noise and increased stability, they lack controllable nonlinearity that is absolutely essential for studying synchronization beyond Kuramoto dynamics. ${ }^{19}$
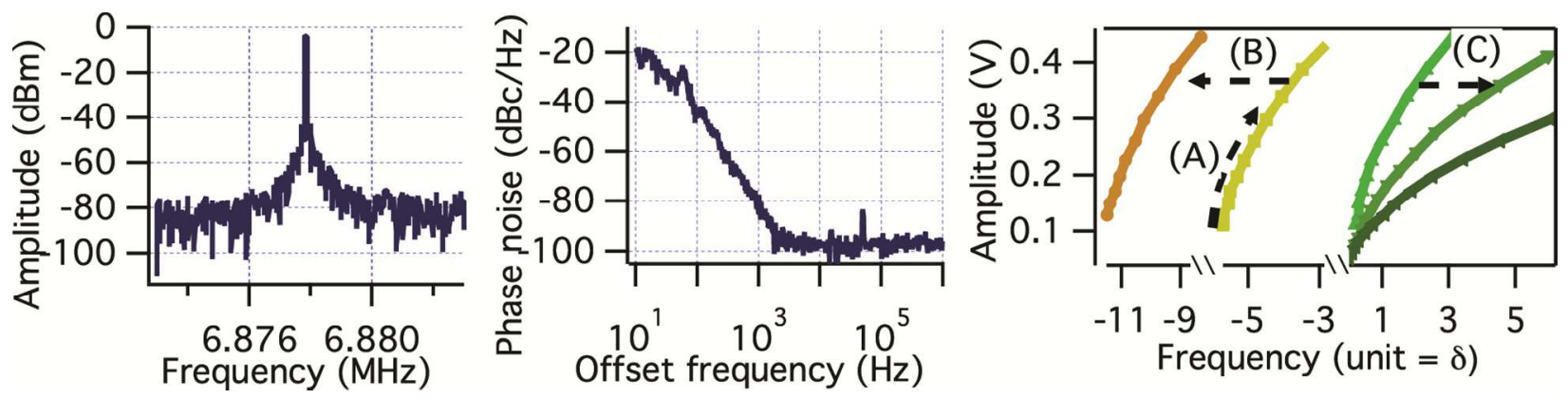

Figure 2. Signal analysis of modular NEMS PCB oscillator. Power spectrum (left) and the phase noise (middle). The power spectrum shows a dominant peak near the NEMS resonant frequency. On the right is the amplitude frequency of an oscillator node under different configuration parameters. In (A), the feedback drive is increased by reducing attenuator AT1 to form the "backbone" curve characteristic to the Duffing nonlinearity. In (B), $V_{D C}=1 \mathrm{~V}$ is applied to tune $\delta_{1}$ by $\sim-5$. In (C), AT2 and AT3 are changed by equal and opposite amounts, which increases the apparent nonlinearity while keeping amplitude constant. 
The key motivations guiding our oscillator-node architecture are: (i) implementation of realtime controllability and programmability; (ii) ease of connectivity, to permit formation of complex network topologies; and (iii) adaptability to use with different classes of NEMS resonators. Many of the parameters and components of the oscillator, including the attenuators, in-loop phase-shifters, oscillator saturation level, and piezoelectric stress of the NEMS, are controllable in real-time with on-board components controlled via the digital link. To form oscillator networks with a centralized control system, all voltages - including the oscillator signal injection input and signal output, as well as its digital control lines, power supplies, ground connections - are accessible through the PCB edge connector, which plugs into the network board. In network operations, such as for the 3-node ring board discussed below, this permits centralized control of all network nodes and edges through the network board. Additional control connections to the individual oscillator boards are not required.

The right panel of Figure 2 shows the flexibility and control of the oscillator node; the frequency-amplitude responses of one oscillator node are displayed for different control parameters. The backbone curves - with display increasing frequency with large amplitude vibration - are the hallmark of the Duffing nonlinearity. By tuning the available parameters, the oscillator nodes can be morphed to different amplitudes, frequencies, and degree of nonlinearity - as required for different investigations. Of interest is the transition " $\mathrm{C}$ " in Fig. 2, where the apparent Duffing nonlinearity of the oscillator increases without a concomitant change in amplitude. This is not trivial, since the Duffing nonlinearity of a NEMS resonator is fixed through device geometry. In our physical implementation, this phenomenon arises because the oscillation $\tilde{A}_{1}$ is referenced at the output of PS/C1 while the Duffing frequency shift 
is referenced to the oscillation in the NEMS as $\tilde{A}_{1}^{\prime}=\epsilon \tilde{A}_{1}$, where $\epsilon$ is a constant depending on the gain of AMP and attenuation at AT3. Therefore, by increasing AT2 and decreasing AT3 by equal amount, $\left|\tilde{A}_{1}^{\prime}\right|$, and thereby the oscillator frequency, are increased while $\left|\tilde{A}_{1}\right|$ remains constant.

To appreciate what is involved in upscaling this architecture to complex network topologies, note that each oscillator node requires fifteen connections. These include the oscillation signal lines, the digital control and digital address buses, and the DC bias lines. All such connections are accessible from the PCB's edge connector and many, including the DC bias levels, can be easily shared across nodes. With deployment of multiple line decoders, all controls of the network board can be multiplexed to a Raspberry-Pi class single-board computer with a single 40-pin ribbon cable. Using software developed for this project, we can perturb the network configuration at sub-millisecond timescales. Given the high fidelity of the extracted signals, the state of the nodal oscillations (their phase and amplitude) can be obtained within a few oscillation cycles, corresponding to a measurement window of a few microseconds. As state evolution follows the slow time scale, of order one millisecond, the requisite bandwidth to follow up to one hundred $2 \mathrm{MHz}$ oscillators, assuming $10 \mathrm{x}$ oversampling, is $10 \times 2 \times(2 \mathrm{MHz}) \times$ $100 \sim 4 G S a / s$. This data rate is readily accessible with modern analog-to-digital (ADC) dataloggers. To summarize, our present implementation of PCB-based nonlinear NEMS networks can easily be scaled to over 100 nodes while retaining full capabilities for network control and state capture. 

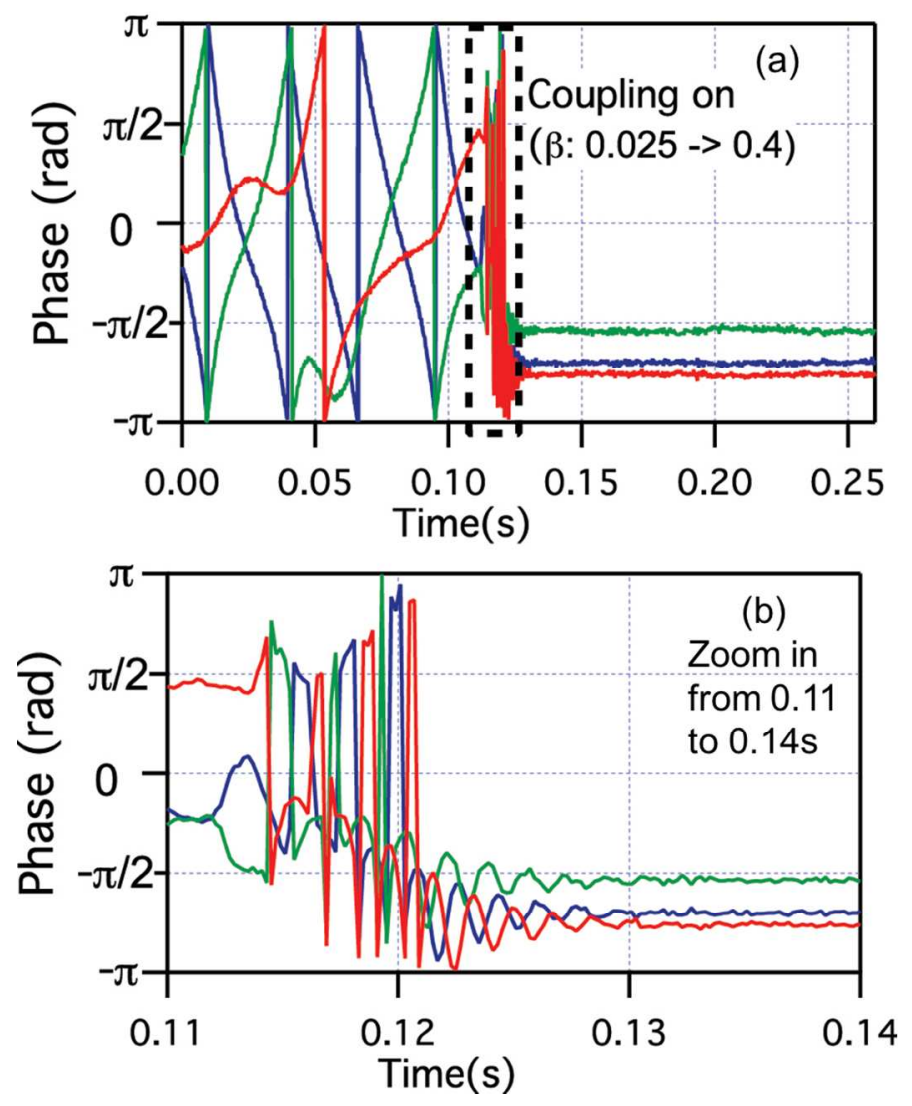

Figure 3: The time-continuous phase difference of 3 oscillators configured as a ring network.

On the top panel (a), at $t=0 \mathrm{~s}$, before the coupling is turned on, the nodes are configured to have

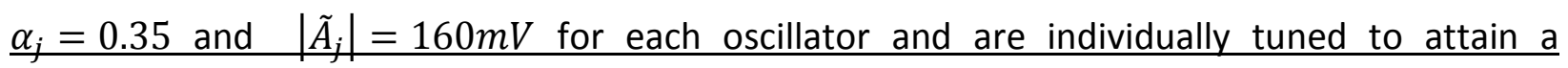
frequency difference of $\Delta \delta=1 \%$. At $t=0.115 \mathrm{~s}$, coupling is switched on. This induces oscillator synchronization after $\sim 15 \mathrm{~ms}$. The detailed evolution of the oscillators during the synchronization process (black dashed box in top panel) is fully captured and displayed in the lower panel (b).

We demonstrate the operation of our nonlinear NEMS network system by implementing a ring network with three oscillator nodes. In a ring-network topology, the nodes are configured in a circle, with each connected to its two nearest neighbors. Mathematically, the coupling is 
defined as $f_{c, j}\left(\tilde{A}_{1}, \tilde{A}_{2}, \tilde{A}_{3}\right)=\frac{i \beta}{2}\left(\tilde{A}_{j+1}-2 \tilde{A}_{j}+\tilde{A}_{j-1}\right)$, where the inter-nodal coupling coefficient, $\beta$, is real. Our 3-node ring network is constructed by plugging 3 NEMS oscillator modules into a ring-network board. As shown in Figure 3, after configuring the control and topology parameters (the Duffing nonlinearity, frequencies, etc.) of the individual oscillator modules, the nodes are run uncoupled for a short interval after which inter-nodal coupling $(\beta)$ is turned on. In this manner, we can capture in real-time the continuous state of the network as it evolves from decoupled oscillators to the fully-synchronized state. The phases of the individual oscillators are extracted from the time records via the Hilbert transformation. To our knowledge, such detailed, real-time observations of the phase of individual oscillators as they transition from the uncoupled to synchronized state have never been presented before in experimental studies. We believe these data, in concert with our theoretical studies, will facilitate discovery of novel network phenomena.

In conclusion, we demonstrate a novel instantiation of nonlinear oscillator networks based on compact, modular, and low-power piezoelectric NEMS oscillators. This architecture readily permits scaling of synchronized, small-scale oscillator networks up to 100 nodes. Examples of intriguing experimental avenues opened by this technology are found in our companion study involving analytical and numerical simulations. ${ }^{29}$ It is noteworthy that the electronic components we employ to construct our modular nodes are fully integratable on-chip via application specific integrated circuit (ASIC) technology. Accordingly, the platform we describe here, constructed from discrete components, serves as a prototype for a next generation of massively-upscaled analog synchronized networks, which can be patterned by co-integration of 
NEMS and CMOS VLSI. ${ }^{41}$ Such co-integrated architecture will make it possible to realize fullycontrollable experimental networks with many thousands of nodes and edges. 


\title{
CORRESPONDING AUTHOR
}

*Michael L. Roukes: roukes@caltech.edu

\begin{abstract}
AUTHOR CONTRIBUTIONS:
All authors contributed to the planning of this research effort. WF and MM contributed equally to device fabrication, measurement system construction, and execution of the experiments. All authors participated in writing the manuscript and have approved the final version.
\end{abstract}

\section{ACKNOWLEDGEMENTS}

We gratefully acknowledge support from the U.S. Army Research Laboratory and the U.S. Army Research Office under Multidisciplinary University Research Initiative Award No. W911NF-13-10340. MLR and MM are also supported by ARO award W911NF13-1-0240. LK was supported by David L. Glackin Memorial Summer Undergraduate Research Fellowship at Caltech. We thank CEA-LETI (Grenoble, France) for providing piezoelectric multilayers enabling this research. We also acknowledge critical support and infrastructure provided for this work provided by the Kavli Nanoscience Institute at Caltech. 


\section{REFERENCES}

1. C. M. Gray, A. K. Engel, P. König and W. Singer, Visual neuroscience 8 (04), 337-347 (1992).

2. E. Davidson and M. Levin, P Natl Acad Sci USA 102 (14), 4935-4935 (2005).

3. I. Dobson, B. A. Carreras, V. E. Lynch and D. E. Newman, Chaos: An Interdisciplinary Journal of Nonlinear Science 17 (2), 026103 (2007).

4. V. Rosato, L. Issacharoff, F. Tiriticco, S. Meloni, S. Porcellinis and R. Setola, International Journal of Critical Infrastructures 4 (1-2), 63-79 (2008).

5. A. E. Motter, S. A. Myers, M. Anghel and T. Nishikawa, Nature Physics 9 (3), 191-197 (2013).

6. M. O. Jackson, Social and economic networks. (Princeton university press Princeton, 2008).

7. D. Helbing, Social self-organization: Agent-based simulations and experiments to study emergent social behavior. (Springer, 2012).

8. J. C. Flack and R. M. D'Souza, P leee 102 (12), 1873-1877 (2014).

9. $\quad$ S. Benz and C. Burroughs, Appl Phys Lett 58 (19), 2162-2164 (1991).

10. S. Kaka, M. R. Pufall, W. H. Rippard, T. J. Silva, S. E. Russek and J. A. Katine, Nature 437 (7057), 389-392 (2005).

11. A. Awad, P. Dürrenfeld, A. Houshang, M. Dvornik, E. lacocca, R. Dumas and J. Åkerman, Nature Physics (2016).

12. M. Bennett, M. F. Schatz, H. Rockwood and K. Wiesenfeld, Proceedings: Mathematics, Physical and Engineering Sciences, 563-579 (2002).

13. A. Marandi, Z. Wang, K. Takata, R. L. Byer and Y. Yamamoto, Nature Photonics 8 (12), 937-942 (2014).

14. S. Y. Shah, M. Zhang, R. Rand and M. Lipson, arXiv preprint arXiv:1511.08536 (2015).

15. E. Gil-Santos, M. Labousse, C. Baker, A. Goetschy, W. Hease, C. Gomez, A. Lemaître, G. Leo, C. Ciuti and I. Favero, Phys Rev Lett 118 (6), 063605 (2017).

16. J. D. Hart, K. Bansal, T. E. Murphy and R. Roy, Chaos: An Interdisciplinary Journal of Nonlinear Science 26 (9), 094801 (2016).

17. T. E. Murphy, A. B. Cohen, B. Ravoori, K. R. Schmitt, A. V. Setty, F. Sorrentino, C. R. Williams, E. Ott and R. Roy, Philosophical Transactions of the Royal Society of London A: Mathematical, Physical and Engineering Sciences 368 (1911), 343-366 (2010).

18. D. P. Rosin, D. Rontani and D. J. Gauthier, Physical Review E 89 (4), 042907 (2014).

19. M. Newman, Networks: an introduction. (Oxford university press, 2010). 
20. A. Pikovsky, M. Rosenblum and J. Kurths, Synchronization: a universal concept in nonlinear sciences. (Cambridge university press, 2003).

21. S. Y. Shah, M. Zhang, R. Rand and M. Lipson, Phys Rev Lett 114 (11), 113602 (2015).

22. M. Zhang, G. S. Wiederhecker, S. Manipatruni, A. Barnard, P. McEuen and M. Lipson, Phys Rev Lett 109 (23), 233906 (2012).

23. M. Bagheri, M. Poot, L. Fan, F. Marquardt and H. X. Tang, Phys Rev Lett 111 (21), 213902 (2013).

24. S. H. Strogatz, Physica D: Nonlinear Phenomena 143 (1), 1-20 (2000).

25. D. E. Nikonov, G. Csaba, W. Porod, T. Shibata, D. Voils, D. Hammerstrom, I. A. Young and G. I. Bourianoff, IEEE Journal on Exploratory Solid-State Computational Devices and Circuits 1, 85-93 (2015).

26. Y. Kuramoto, in International symposium on mathematical problems in theoretical physics (Springer, 1975), Vol. 39, pp. 420-422.

27. M. H. Matheny, M. Grau, L. G. Villanueva, R. B. Karabalin, M. Cross and M. L. Roukes, Phys Rev Lett 112 (1), 014101 (2014).

28. R. Lifshitz and M. Cross, Review of nonlinear dynamics and complexity 1, 1-52 (2008).

29. J. Emenheiser, A. Chapman, M. Pósfai, J. P. Crutchfield, M. Mesbahi and R. M. D'Souza, Chaos 26 (9), 094816 (2016).

30. R. B. Karabalin, L. Villanueva, M. Matheny, J. E. Sader and M. L. Roukes, Phys Rev Lett 108 (23), 236101 (2012).

31. R. Karabalin, S. Masmanidis and M. Roukes, Appl Phys Lett 97 (18), 183101 (2010).

32. R. Karabalin, M. Matheny, X. Feng, E. Defaÿ, G. Le Rhun, C. Marcoux, S. Hentz, P. Andreucci and M. Roukes, Appl Phys Lett 95 (10), 103111 (2009).

33. L. Villanueva, E. Kenig, R. Karabalin, M. Matheny, R. Lifshitz, M. Cross and M. Roukes, Phys Rev Lett 110 (17), 177208 (2013).

34. K. Ekinci and M. Roukes, Rev Sci Instrum 76 (6), 061101 (2005).

35. M. Matheny, L. Villanueva, R. Karabalin, J. E. Sader and M. Roukes, Nano letters 13 (4), 1622-1626 (2013).

36. L. G. Villanueva, R. B. Karabalin, M. H. Matheny, E. Kenig, M. C. Cross and M. L. Roukes, Nano letters 11 (11), 5054-5059 (2011).

37. X. Feng, C. White, A. Hajimiri and M. L. Roukes, Nature nanotechnology 3 (6), 342-346 (2008).

38. P. Horowitz, W. Hill and I. Robinson, The art of electronics. (Cambridge university press Cambridge, 1980).

39. Y.-W. Lin, S. Lee, S.-S. Li, Y. Xie, Z. Ren and C.-C. Nguyen, leee J Solid-St Circ 39 (12), 2477-2491 (2004). 
40. D. Howe, D. Allan and J. Barnes, from the website of NIST as was found on 10, 3 (2005).

41. J. Arcamone, M. Savoye, G. Arndt, J. Philippe, C. Marcoux, E. Colinet, L. Duraffourg, T. Magis, M. Laurens and A. Monroy-Aguirre, Electron Devices Meeting (IEDM), 2012 IEEE International (2012). 